\title{
Attochemistry: Is Controlling Electrons the Future of Photochemistry?
}

\author{
Isabella C.D. Merritt, Denis Jacquemin, and Morgane Vacher* \\ Université de Nantes, CNRS, CEISAM, UMR 6230, F-44000 Nantes, France \\ E-mail: morgane.vacher@univ-nantes.fr
}




\begin{abstract}
Controlling matter with light has always been a great challenge, leading to the ever-expanding field of photochemistry. In addition, since the first generation of light pulses of attosecond ( 1 as $\left.=10^{-18} \mathrm{~s}\right)$ duration, a great deal of efforts has been devoted to observing and controlling electrons on their intrinsic timescale. Because of their short duration, attosecond pulses have a large spectral bandwidth populating several electronically excited states in a coherent manner, i.e., an electronic wavepacket. Due to interference, such a wavepacket has a new electronic distribution implying a potentially different and totally new reactivity as compared to traditional photochemistry, and leading to the novel concept of "attochemistry". This nascent field requires the support of theory right from the start. In this Perspective, we discuss the opportunities offered by attochemistry, the related challenges, as well as the current and future state-of-art developments in theoretical chemistry needed to model it accurately.
\end{abstract}

\title{
Graphical TOC Entry
}

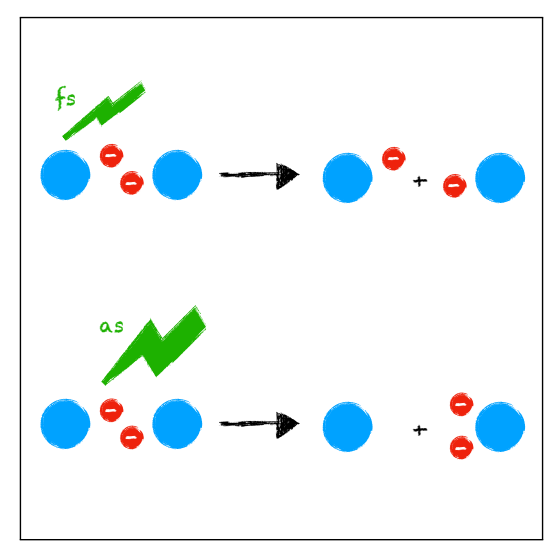


The development of femtosecond ( $\left.1 \mathrm{fs}=10^{-15} \mathrm{~s}\right)$ pulses in the $1980 \mathrm{~s}^{1}$ allowed for the study, and later control, of nuclear motions which occur naturally on the femtosecond timescale. Modulation of amplitudes and phases of the spectral components in a femtosecond photoexcitation pulse has been explored heavily to coherently populate different vibrational states and form nuclear wavepackets: the resulting constructive or destructive interferences can lead to a control of the chemical reactivity. ${ }^{2-7}$ An illustrative example is the $20 \%$ increase of the yield of the photoisomerisation of retinal in bacteriorhodopsin through harnessing of vibrational coherences. ${ }^{8}$ Pulses could further be optimized to give the desired products using the so-called optimal control theory (OCT), allowing formation of laser pulses of highly complex forms. ${ }^{9}$ OCT has been used to predict complex UV laser pulses which will populate certain vibrational states of a single electronic state before passing through a conical intersection (CI), and by doing so control chemical reactivity. ${ }^{10} \mathrm{CIs}$ are now well-recognized as fundamental parts of photochemical reactions, ${ }^{11-16}$ acting as funnels for electronic population from an upper excited state to a lower electronic state and determining subsequent evolution of the molecular system. In this context, de Vivie-Riedle et al. proposed to extend OCT to the coherent population of electronic states, optimizing the pulses used to generate electronic wavepackets. ${ }^{9}$

Since the first production of attosecond pulses 20 years ago, ${ }^{17,18}$ attosecond science has grown exponentially. The synthesis of such pulses now routinely carried out in laboratories using high harmonic generation marks a turning point in the generation of electronic wavepackets. ${ }^{19}$ Indeed, attosecond pulses match the natural timescale of electronic motion in molecules, paving the way to the direct observation and control of the electrons. ${ }^{20-22}$ If chemistry is defined as the science of breaking and forming chemical bonds, then controlling the electrons responsible for the bonds is the most fundamental way of doing chemistry. ${ }^{23,24}$ This is the true goal of attochemistry - to directly control bonding by steering the attosecond electron dynamics in molecules. Attochemistry relies on the following central concept: pulses of extremely short duration have also a large spectral bandwidth, and 
can therefore be used to coherently populate several electronic excited states of a molecule, referred to as an "electronic wavepacket". Because of interference phenomena, the electronic wavepacket has a new electronic distribution and potentially a new chemical reactivity. Attochemistry, coming from the synergy between the two fields of attosecond science and photochemistry, aims at exploring the a priori very broad spectrum of photochemical reactions that could be induced by an attosecond pulse, compared to a "traditional" femtosecond photoexcitation of a single electronic excited state. We distinguish here photochemistry - when the absorption of light causes a chemical reaction - from photophysics - otherwise. In the same manner, "attochemistry" is used here to refer to the induction of a chemical reaction, i.e., the formation of a different chemical compound, by an attosecond pulse.

One of the most important concepts to keep in mind in coherent control schemes is that, in order to use a superposition of states to control reactivity, both a "pump" and "probe" event are required. It is not enough to simply form an initial coherent superposition of states. There must be a second dynamical event which couples the states back together, in order to use the coherence between the states to control the outcome of the reaction. This is a general concept, frequently discussed in the context of using coherent nuclear wavepackets to control reactions, ${ }^{3,5}$ and the theory is equivalent for coherent electronic superpositions of adiabatic states. This was phrased simply and understandably by Lépine et al.: "on absorption of a single photon, each populated eigenstate yields a given product, with electronic coherence between states playing no role unless a second probe step is employed to "recouple" the states and induce coherent-dependent dynamics". ${ }^{21}$ The "probe" interaction does not need to be a second light pulse, but can be any dynamical event - such as electron-ion recollision, coupling to a bath that exchanges energy with the system, or coupling of the electronic states with the nuclear degrees of freedom as seen at CIs. The last of these is arguably the most interesting to chemists, CIs playing pivotal roles in photochemical processes. ${ }^{14-16}$

"Charge-directed reactivity" is used in the literature as a general term to refer to chemical 
reactions induced by a superposition of electronic states. ${ }^{25}$ This nomenclature comes from the experiments of Weinkauf et al. performed several years before attosecond pulses were first produced. ${ }^{26,27}$ After locally ionizing one end of a peptide chain with nanosecond UV pulses, photofragmentation by subsequent photon absorption occurred at the other end of the chain even at energies close to the dissociation threshold. ${ }^{26,27}$ This was inexplicable within the classical RRKM model of dissociation: the low excess energy should be distributed across vibrational modes over the whole molecule, and fragmentation should be prevented by the high entropy barrier to energy localization. The observation of dissociation despite this prompted discussions on the idea of an alternative, purely electronic, mechanism which localizes charge before the excess energy is dissipated. This was additionally supported by the lack of dependence of the localized dissociation on the size of the molecule. ${ }^{25,28}$

The two main schemes to form a coherent superposition of multiple electronic states are: (1) triggering excited state dynamics on a single state as in "traditional" photochemistry, and then coupling several electronic states using a "control" pulse (typically an IR laser pulse) just before the standard excited state dynamics reaches the CI; ${ }^{9,29-31}$ and (2) exciting directly to a superposition of electronic states at the Franck-Condon geometry using an attosecond pulse. ${ }^{27,32-34}$ In both cases, manipulation of the exact composition of the created electronic wavepacket (i.e., relative weights and phases between the electronic states) is expected to allow control of the induced nuclear motions and the chemical reactivity. Due to the recent emergence of the attoscience field, early schemes proposed for the control of nuclear motion via electronic superpositions typically relied on control IR pulses. ${ }^{9,29,35}$ In the following, we first provide examples of such studies which, although not relying on attosecond photoexcitation, provide valuable knowledge on the chemical reactivity induced by an electronic wavepacket. We then illustrate the current state-of-the-art of "true" attochemistry, as well as the challenges needed to be addressed in the future of this nascent field.

There have been several theoretical and experimental studies relying on a control IR pulse to create a specific electronic wavepacket at some particular time after initial pho- 
toexcitation to influence the induced nuclear dynamics. More precisely, the idea is to use the carrier-envelope phase (CEP) of a phase-stabilized IR pulse to modulate the relative phase within the electronic wavepacket. The CEP of a pulse is defined as the phase difference between the pulse envelope and the electric field waveform as shown in Figure 1a. It was first theoretically predicted to allow for the modulation of the asymmetry of ionisation of diatomic molecules, e.g., the single-electron diatomic ions $\mathrm{H}_{2}{ }^{+}, \mathrm{HD}^{+}$and $\mathrm{D}_{2}{ }^{+} .36,37$ These simulations described the coupled electron-nuclear dynamics by solving the full timedependent Schrödinger equation numerically on a grid, with a single nuclear coordinate and with one ${ }^{37}$ or two ${ }^{36}$ dimensions for the single electron.

The effect of manipulating the phase difference between electronic states has first been observed in the experiments of Kling et al. combined with the simulations by the group of de Vivie-Riedle, in the context of the strong field dissociative ionisation of diatomic $\mathrm{D}_{2}$ and its isotopologues. ${ }^{29,30,38-40}$ Dissociative ionisation using strong IR laser fields through the recollision excitation channel investigated in these experiments is a complex physical process, typically described using the three-step mechanism shown in Figure 1b: (1) laser induced tunnel ionisation of the neutral molecule; (2) recollision excitation of the ion to excited state(s); and (3) dissociative ionisation and laser-induced coupling of ionic excited states. All three steps are both driven and influenced by the IR laser field. Coherent control comes into play in the coupling together of excited states by the IR pulse in step (3), building up a superposition with phase difference controlled by the CEP of the laser pulse. This superposition of states triggers electron dynamics consisting of the electron oscillating between the two atoms of the molecule. As the coherent wavepacket evolves in time, at some time the distance between the two atoms becomes too large for the electron oscillation to continue and the charge is localized - the dissociation acts as the "probe" interaction. As a result, directional emission of the $\mathrm{D}^{+}$ion is measured, with an asymmetry dependent on the CEP of the laser used - at CEP values of 0 and $\pi$, identical and opposite symmetry is seen in the emission of $\mathrm{D}^{+}$ions since the electronic superpositions formed at these CEP values 
are of opposite sign, see Figure 1c. ${ }^{29,30}$

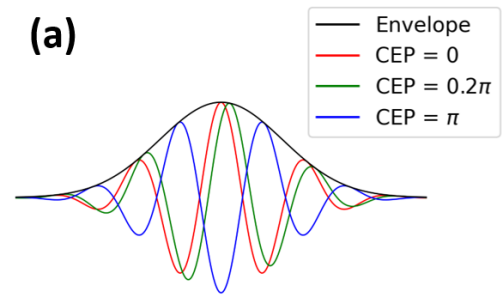

(b)
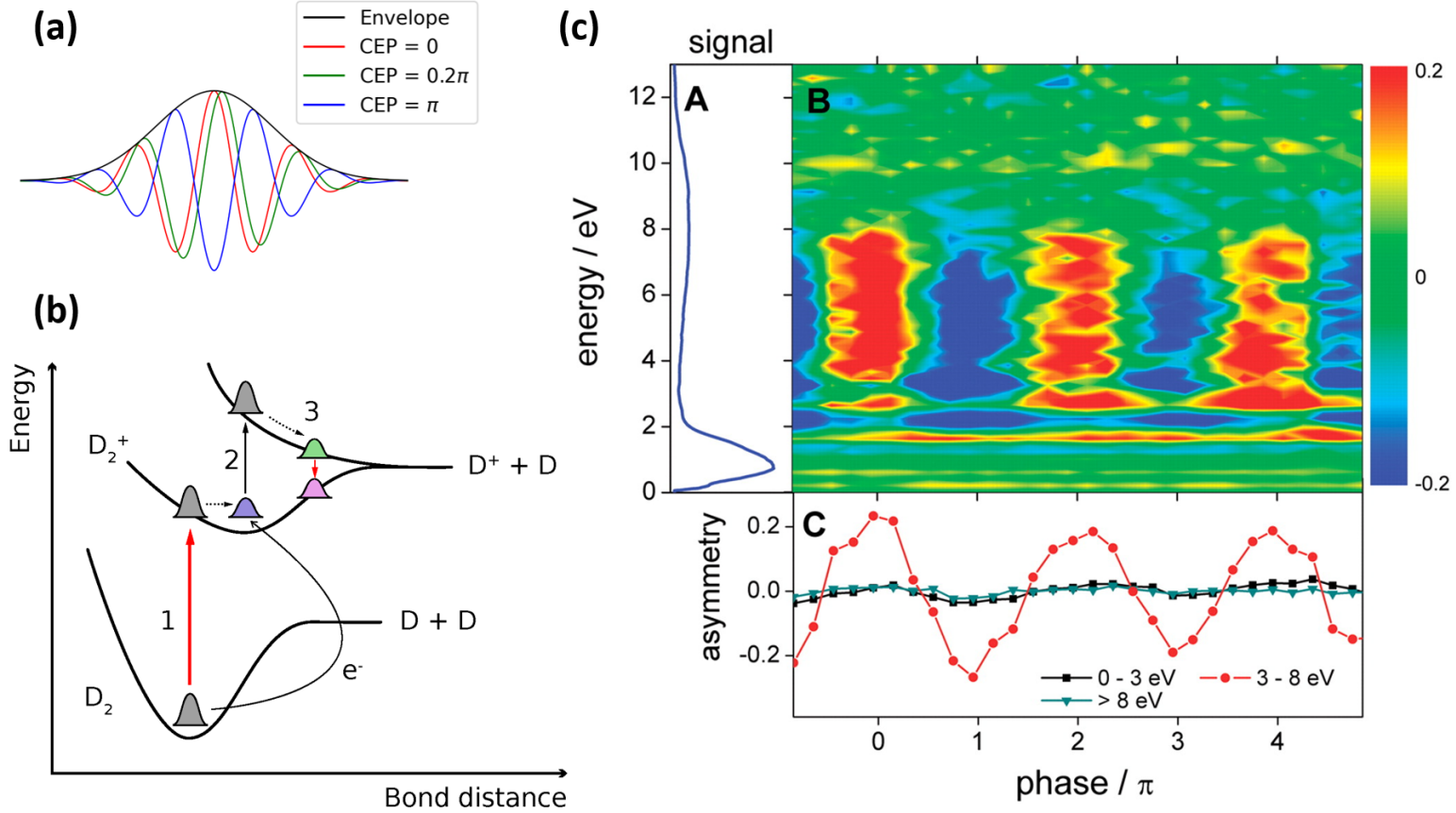

Figure 1: (a) Visual representation of the carrier-envelope phase (CEP) of a few-cycle pulse. (b) Mechanism for dissociative ionization through the recollision excitation channel. (c) Measured and predicted dependence of the asymmetry in directional ion emission in $\mathrm{D}_{2}$ dissociative ionization on changing the CEP of the IR laser used. From [Kling et al, Science 2006, 312, 5771, 246-248]. Reprinted with permission from AAAS.

These experiments on $\mathrm{D}_{2}$ dissociative ionisation were the first examples of using lightfields to trigger specific electron dynamics in a molecule through formation of a particular electronic wavepacket, allowing control of the subsequent nuclear motion. However, one of the main issues with CEP control using strong-field laser pulses is that the pulse can influence the dynamics in other ways. Indeed, all three steps in the dissociative ionization depend on the IR pulse's electric field, ${ }^{38}$ resulting in a theoretically very complex system making it challenging to untangle the different contributions to the CEP dependence of the asymmetry. In addition, one electron diatomics remain proof of principle models only. If the single electron and single nuclear coordinate allow for fully quantum theoretical descriptions of both electronic and nuclear degrees of freedom, whether light-fields could be applied to control chemical reactions in multi-electron and larger molecules remained to be investigated. 
Following their studies on $\mathrm{D}_{2}$, subsequent experiments were carried out using an analogous CEP control scheme on both $\mathrm{CO}^{41}$ and $\mathrm{DCl} .{ }^{42}$ To describe multi-electron systems, the associated simulations relied on quantum chemistry methods. CEP dependent asymmetric dissociation was observed in both compounds. However, thanks to comparisons between measured and simulated data, the CEP observed asymmetry in each of these two systems was attributed to different mechanisms to that described for $\mathrm{D}_{2}$. For $\mathrm{CO}$, the superposition of electronic states is instead formed by the recollision step (2) - the phase differences inside the initial electronic wavepacket are not controlled by the CEP of the laser pulse. ${ }^{38,41}$ The strong field of the IR pulse then influences the electron dynamics which arises from this recollision formed superposition in a way controlled by the CEP. For DCl, another mechanism entirely explains the CEP dependence, relating to the initial ionisation step (1). Adjusting the CEP of pulse can control the orientation of the formed $\mathrm{DCl}^{+}$, and consequently the asymmetry of the charged fragment emission; ${ }^{43}$ a mechanism independent of electron dynamics. ${ }^{42}$

Extending the concepts explored in CEP control of diatomic dissociation theoretically to larger molecular systems, de Vivie-Riedle et al. suggested a superposition of electronic states could be built up near to a CI and used to steer chemical reactions. ${ }^{9,30}$ Near to these points, the Born-Oppenheimer approximation breaks down due to the strong non-adiabatic couplings, and the energy gap between electronic states becomes small so that the electron dynamics associated with the superposition slows down. In other words, the areas around CIs can be seen as regions where the electron dynamics slows to be on the timescale of nuclear dynamics, inducing much larger coupling between the electron and nuclear motions. ${ }^{21,30}$ Using quantum coupled electron-nuclear dynamics on an analytical model system consisting of three electronic states and two nuclear coordinates, ${ }^{44}$ they were able to modulate the final $\mathrm{S}_{1}: \mathrm{S}_{2}$ population ratio after passage through the CI by altering the phase of the superposition of states using the CEP of the IR pulse. ${ }^{45}$

This scheme has been further explored for more realistic systems. ${ }^{46,47}$ In a study on both the previously studied analytical model system ${ }^{44}$ and uracil for which the nuclear dynamics 
is described quantum mechanically in a reduced space of two nuclear coordinates, ${ }^{46}$ also incorporating the IR pulse into the dynamics, two mechanisms were identified to play a role in CEP control of population ratio. At low IR field strengths the mechanism predicted by the earlier control scheme dominates, with the expected periodicity of $2 \pi$ typical to phase dependent processes. At higher IR field strengths the temporal asymmetry of the IR pulse results in a $\pi$ periodicity, and CEP control is due to the overall shape of the pulse changing with CEP, and not the coherence between states.

In a separate study, the same group investigated the effect of applying an IR pulse to influence the passage of the first excited state through a CI of the $\mathrm{NO}_{2}$ cation. ${ }^{47}$ The IR pulse builds up a superposition before passage through the CI as in their earlier proposals, ${ }^{9,30}$ but additionally influences the electron dynamics during and after passage through the CI and interferes with the intrinsic electron dynamics induced by the CI without any IR pulse. The interaction of the IR pulse with the system is non-trivial, and uncoupling different effects is complicated. In their simulations the controllability of nuclear and electron dynamics using the CEP of the IR pulse was demonstrated: by projecting out different observables two mechanisms of CEP control were observed as seen in their earlier paper, ${ }^{46}$ with periodicities of $\pi$ and $2 \pi$. The nuclear asymmetry relating to the derivative coupling vector (see Figure 2) was characterised by the $2 \pi$ periodicity typical of an interference process. ${ }^{48-50}$

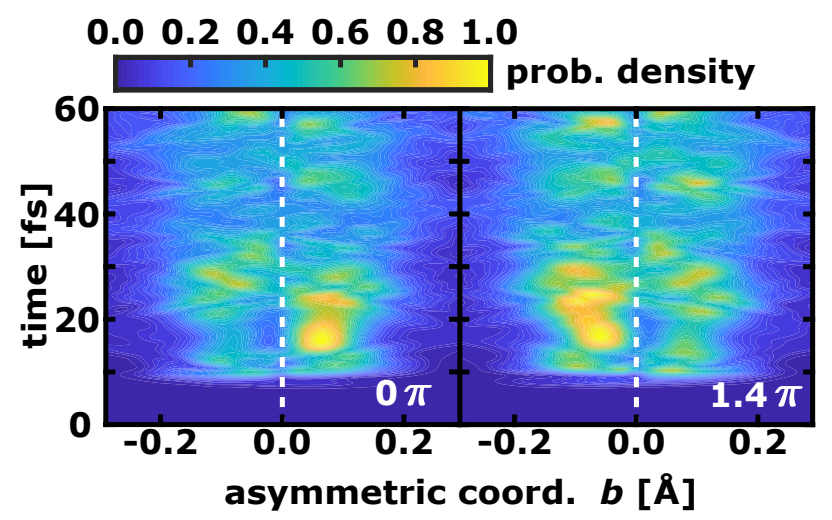

Figure 2: Time evolution of the nuclear probability density on the adiabatic ground state surface along the derivative coupling coordinate (asymmetric coordinate $b$ ) in the presence of a few-cycle IR pulse with a CEP of $0.0 \pi$ (left) and $1.4 \pi$ (right). Reproduced from [Schnappinger et al, J. Chem. Phys. 154, 134306 (2021)], with the permission of AIP Publishing. 
Using a single CEP controlled strong IR pulse, Remacle and coworkers demonstrated the possibility to steer the nuclear dynamics to specific dissociation products in the LiH diatomic molecule, and further to control the dissociation yields. ${ }^{51}$ Assuming aligned molecules and using also the polarization of the IR pulse, coherent electronic wavepackets with different ionic characters could be built, inducing different fragmentation channels, see Figure 3 . The same group also studied coupled electron-nuclear dynamics in the $\mathrm{N}_{2}$ molecule. ${ }^{52}$

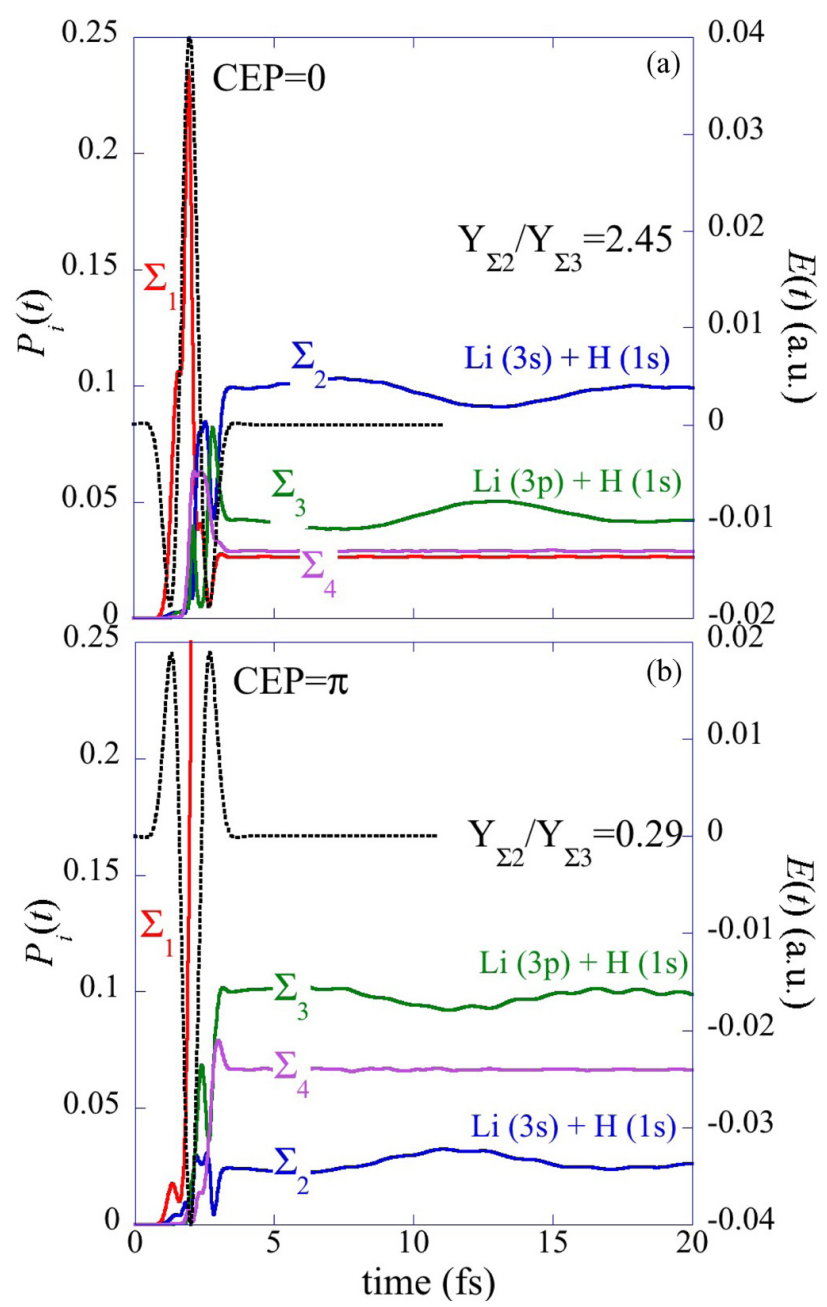

Figure 3: Effect of the CEP on the populations of the different electronic states, $P_{i}(t)$, with (a) $\mathrm{CEP}=0$ and (b) $\mathrm{CEP}=\pi$. Reprinted figure with permission from [Nikodem et al, Phys. Rev. A, 95, 053404 (2017)]. Copyright 2017 by the American Physical Society.

The theoretical studies summarized above treat quantum mechanically the nuclear motion by solving numerically (on a grid) the time-dependent Schrödinger equation, at the expense of taking into account a few nuclear coordinates only. Using Quantum-Ehrenfest 
dynamics, ${ }^{53}$ Robb et al. studied such coupled electron-nuclear dynamics under the influence of a control IR pulse in the allene radical cation in a more approximate way but taking into account the 15 nuclear degrees of freedom of that compound. ${ }^{54}$ In the Quantum-Ehrenfest method, the nuclear wavepacket is described by a linear combination of Gaussian basis functions. The amplitudes of the Gaussian functions are determined quantum mechanically by solving the time-dependent Schrödinger equation. Each Gaussian function moves however independently and classically along a trajectory. The overall molecular wavepacket is thus built as an exact solution expressed in a basis of approximate trajectories. In contrast to the ab initio multiple spawning method where each trajectory is determined by a single adiabatic state, ${ }^{55,56}$ in the Quantum Ehrenfest method, each trajectory is determined by an Ehrenfest mean-field potential. The authors investigated the effect of an IR control pulse on the dynamics of an already established electronic superposition passing through a CI in the allene radical cation. Their results showed that the application of such a control pulse could strongly impact the electron dynamics through the CI, and the nuclear dynamics, significantly altering the torsional motion on a much longer timescale than the length of the IR pulse itself.

To summarize these results of charge-directed reactivity obtained with an IR control pulse, the possibility of tailoring superpositions of states to control electronic motion and the resultant chemical changes has been clearly highlighted. However, the complex strongfield physics induced by the strong field IR pulses complicates this CEP laser control scheme. It is non-trivial to separate the effects of the superposition formation from those of the the laser field itself on the electron dynamics. Using an attosecond pulse to directly excite a superposition of states at the Franck-Condon geometry therefore appears as an attractive alternative. We underline that since attosecond pulses are typically high energy, they very often lead to an ionisation of molecules and not only excitation. ${ }^{35,57,58}$ As a result, most experimental and theoretical studies carried out so far have followed ionisation. ${ }^{34,59-64}$ The concept is however general for any superposition of electronic states. Indeed, the formation 
of electronic superpositions in neutral molecules using UV/vis excitation could provide a more gentle way to control molecules, by directing bound electrons to control photochemical reactions while avoiding photofragmentation. Controlling bound electrons without ionization using attosecond pulses is certainly challenging, since the pulses required are harder to generate being few-cycle or less; but the generation of half-cycle attosecond pulses in the UV-vis range, ${ }^{65}$ and few-cycle deep UV sub-2-fs pulses, ${ }^{66}$ have been reported. In particular, a 2019 combined theoretical and experimental work investigated the dynamics induced upon population of an electronic wavepacket made of the electronic ground state and the first excited state of neutral $\mathrm{O}_{3}$ molecule using a deep UV pulse. ${ }^{67}$ By modifying the attosecond pulses used for excitation, the initial electronic superposition formed could often be tuned. ${ }^{33,68-71}$

Historically, at the same time as the first attosecond pulses were generated, the observations by Weinkauf et al. served as the catalyst for the development by Cederbaum et al. of their charge migration theory, ${ }^{32,72}$ whereupon electron correlation drives ultrafast rearrangement of the electron cloud on an attosecond timescale. ${ }^{59}$ This differs from charge transfer, where the movement of electrons is due to the motion of nuclei and occurs on much longer timescales. ${ }^{73}$ The electron dynamics is due to the electronic wavefunction formed upon ionization not being an eigenstate of the cation. Rather, it can be expressed as a superposition of several cationic eigenstates which leads to charge oscillation across the molecule. The period of oscillations $T$ is inversely proportional to the energy gap between states, ${ }^{21,30,63}$

$$
T=\frac{h}{E_{2}-E_{1}}
$$

The charge, initially localized at one end of the molecule, could later be trapped at the opposite end of the molecule by nuclear motion, determining where photofragmentation takes place.

In the earliest descriptions of attosecond electron dynamics, the nuclei were assumed 
to be stationary as they were expected to move on timescales much slower than the electron dynamics. ${ }^{74}$ Using this fixed single-geometry approach avoids the need for expensive descriptions of the coupling between electron and nuclear motion in simulations, and detailed quantum descriptions of electronic motion can be more easily applied. Initial attosecond theoretical studies neglected nuclear coordinates and described the electron dynamics quantum mechanically, solving the electronic time-dependent Schrödinger equation by discretizing time and in the basis of electronic eigenstates. The latter were typically calculated with quantum chemistry methods such as TD-DFT (Time-Dependent Density Functional Theory), ${ }^{75,76}$ ADC (Algebraic Diagrammatic Construction), ${ }^{77,78}$ CASSCF (Complete Active Space Self-Consistent Field), ${ }^{79}$ etc. Such models predict long-lived oscillations in the electron dynamics. ${ }^{32,34,68,80}$ The oscillating electronic density comes from oscillations in the coherences between electronic eigenstates, while the populations of the latter stay constant. However, simulations including the nuclear motion approximately on molecular cations of for instance benzene, toluene, glycine, and 2-phenyl-ethyl-amine demonstrated that nuclear motion can affect both the nature and timescale of the oscillating motion in the electronic density. ${ }^{73,81,82}$ This is partly due to the non-adiabatic coupling between electrons and nuclei, and the resulting transfer of population between the electronic eigenstates. The amplitude of these effects is of course system dependent and can become significant after $2-3$ fs only. These results were obtained using single Ehrenfest trajectories, i.e., the nuclei are moving classically on mean-field potentials taking into account all nuclear coordinates. Later simulations on xylene and polycyclic norbornadiene cations including ensembles of Ehrenfest trajectories predicted a fast electronic decoherence because of the nuclear wavepacket width. ${ }^{64,83}$ The fast electronic decoherence occurring on the femtosecond timescale was then confirmed on other molecules by one of us using the DD-vMCG (Direct-Dynamics variational Multi-Configuration Gaussian) method, ${ }^{62}$ and by other groups using the MCTDH (Multi-Configuration Time-Dependent Hartree) method. ${ }^{50,84-86}$ Both of these approaches treat quantum mechanically the nuclear motion. While the latter typically requires the de- 
velopment of a model Hamiltonian, a reduced number of nuclear coordinates being often taken into account, ${ }^{87}$ the former calculates the potential energy surfaces on-the-fly aiming at describing all nuclear coordinates. ${ }^{88}$ In the DD-vMCG method, as in Quantum-Ehrenfest, the molecular wavepacket is described using a basis of Gaussian functions. ${ }^{89}$ Here, the Gaussian functions move quantum mechanically obeying the time-dependent Schrödinger equation. This ensures a faster convergence to the in principle exact result. The coherence lifetime was shown to depend on molecular properties such as the non-adiabatic coupling strength, the relative shape of the coupled state potential energy surfaces, and the nuclear wavepacket widths. ${ }^{50,62,63,86}$

The idea of attosecond charge-directed reactivity - using an attosecond pulse to directly control the formation and breaking of chemical bonds by controlling electron rearrangement in molecules - unsurprisingly greatly inspires chemists. One can imagine that by controlling the initial electronic wavepacket created, one could control the electron dynamics that occurs following excitation. In order to use electron dynamics to control nuclear motion though, the coherence must be preserved at the "probe" interaction: therefore, the "probe" interaction must occur early enough after the formation of the electronic superposition. The first challenge of attochemistry is the following: obtaining an electronic coherence (or a "legacy") living long enough to affect the outcome of a photochemical reaction. Assuming this, the charge distribution induced by the electron dynamics triggers the desired nuclear dynamics which evolves over much longer timescales. ${ }^{71}$ We wish to emphasize that shooting an attosecond pulse on a molecule and exciting "randomly" a high number of electronic excited states of diverse nature is unlikely to lead to a significant effect on the photochemical process of interest. To be effective, it is necessary to target a specific and carefully chosen electronic wavepacket consisting of probably a few electronic eigenstates only. Ideal spectral widths are then a few $\mathrm{eV}$, corresponding to pulse durations of hundreds of attoseconds to few femtoseconds. Limiting the number of electronic eigenstates populated coherently is neither due to theoretical nor experimental limitations. In 
fact, the interference between many electronic eigenstates of diverse nature often results in a hole density delocalized over the whole molecule; the modulation of which being small. The chances to induce control from such an electronic density is logically very low. The second challenge of attochemistry is to determine: what electronic wavepacket (i.e., what complex amplitudes, weights and phases in the superposition of states) will either induce one otherwise impossible chemical reaction, or largely enhance the yield of one possible-yet-inefficient photochemical process? The follow-up challenge is then to design an experiment and a pulse which excites such a wavepacket. Through control of the attosecond "pump" excitation that forms the electronic wavepacket, we can tailor both the states and their respective populations which make up the wavepacket, as well as the relative phases between these states. Controlling the states occupied is a relatively simple theoretical concept - the energy range of the pulse determines which excitations can occur and thus the resulting states populated. The exact energy range covered can be selected by the formation method of the pulse and its duration. ${ }^{17,18,57,65,66}$ The other "control knob" for the initial electronic wavepacket is the phase difference between the coherently populated states, suggested to be controllable for attosecond and few-femtosecond pulses through pulse parameters such as the chirp, or by use of $\pi$ pulses. ${ }^{71}$ The polarization of the laser field can also be a useful parameter to influence the composition of the coherent superposition of states. ${ }^{90}$ To tackle the above challenges, attochemistry requires theoretical support right from the start.

The previous CEP experiments demonstrated that it is possible to influence nuclear motion using an electronic superposition. For the general design of successful charge-directed reactivity experiments, a systematic study and deep understanding of the effect the electronic superposition has on the electronic density and the subsequent nuclear motion are necessary. The impact a superposition of electronic states has on the initial nuclear motion in the CI branching space upon leaving the CI has been theoretically investigated in several works. ${ }^{48-50}$ More precisely, the effect of superposition ratio and phase was investigated theoretically using Ehrenfest dynamics in two contributions by Robb et al. dedicated to toluene ${ }^{48}$ and benzene ${ }^{49}$ 
cations. Assuming valence photoionisation, the formed electronic wavepacket is defined as a superposition of the two lowest-energy eigenstates of the cation, by

$$
\Psi=\cos (\theta / 2) \psi_{0}+\sin (\theta / 2) e^{i \phi} \psi_{1}
$$

$\theta$ defining the relative weight of the two electronic states and $\phi$ the relative phase. In toluene, the equilibrium geometry of the neutral species corresponds to a geometry slightly displaced from the CI in the cationic species whereas in benzene the superposition is formed directly on the CI seam. The study on toluene demonstrated that changing the relative weight of an in-phase superposition of states could control the direction in the branching space as seen in Figures 4a and b, with an equal ratio of states resulting in initial nuclear motion orthogonal to that of the pure electronic states, along the derivative coupling vector (Figure $4 \mathrm{~b})$. By controlling the phase of an equally weighted superposition, the direction and velocity along the derivative coupling vector could be controlled (Figure 4b). ${ }^{48}$ The study on benzene extended this investigation to a complex rotation of diabatic states and demonstrated that by modulating ratio and phase concurrently both direction and velocity in the full branching space could be controlled (Figure 4c). The group of Remacle studied similar structural rearrangements due to Jahn-Teller effects upon sudden ionisation of methane. ${ }^{91}$ Those simulations used a quantum mechanical treatment of nuclear motion in a reduced space of two nuclear coordinates.

In a separate study one of us investigated, using the Ehrenfest method again, the coupled electron and nuclear dynamics of modified bismethylene-adamantane (BMA) cations. ${ }^{63}$ This work not only predicted the dephasing of electron dynamics due to the nuclei, but showed that the initial non-stationary wavepacket formed determines the initial nuclear dynamics. When initiating dynamics with a superposition of states, an asymmetric stretching in the terminal methyl groups of the BMA cations was observed in the simulations, while population of a single electronic state led to symmetric stretching only. Controlling the phase of the 

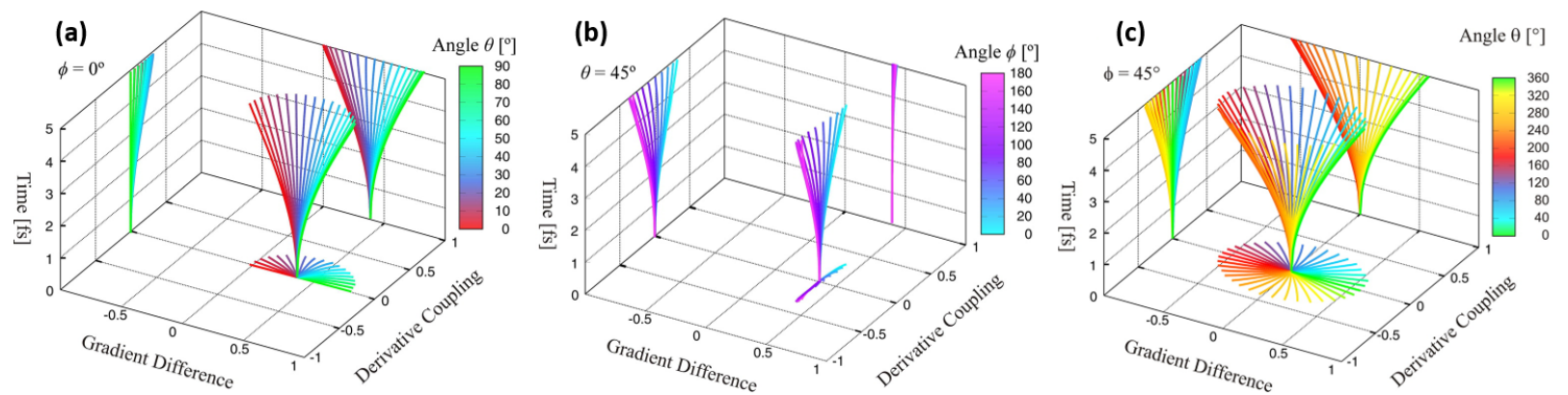

Figure 4: Nuclear trajectories in the branching space initiated with different initial electronic superpositions in toluene $(\mathrm{a}, \mathrm{b})$ and benzene (c). (a) Varying the relative weight of two in phase $(\phi=0)$ states. (b) Varying the phase difference between two equally weighted $(\theta=45)$ states. (c) Varying the relative weight of two out of phase states, with a phase difference of $\phi=\pi / 4$. Panels (a) and (b) reprinted with permission from [Vacher et al, J. Phys. Chem. A 2015, 119, 21, 5165-5172]. Copyright 2014 American Chemical Society. Panel (c) reprinted with permission from [Meisner et al, J. Chem. Theory Comput. 2015, 11, 7, 3115-3122]. Copyright 2015 American Chemical Society.

superposition of states through the $\phi$ parameter led to the differences in the direction and timing of the asymmetric stretching shown in Figure 5. This demonstrated the possibility of controlling the initial nuclear motion - which bond stretches more and by how much by manipulating the composition of the initial electronic wavepacket formed through the relative weight $\theta / 2$ and phase $\phi$ : i.e., a step towards charge-directed reactivity.

It is essential to keep in mind two major limitations of these theoretical studies: (1) the Ehrenfest method does not allow the nuclear wavepackets on the different electronic states to move in different directions; (2) the trajectories simulated to represent as an ensemble the spatial delocalization of nuclear wavepackets are not coupled. Both of these may affect electronic decoherence and thus the predicted control of nuclear motion. A more accurate dynamics method would be desirable to describe such coupled electron and nuclear dynamics. Building on these findings, applying a two-state two-mode linear vibronic coupling model Santra et al. investigated how the strength of non-adiabatic coupling and the position of the CI relative to the Frank-Condon point could affect the controllability of the initial nuclear dynamics. ${ }^{50}$ Using an equal superposition of states and varying the phase, they obtained the same result as the Ehrenfest studies ${ }^{48,49}$ - that the phase of an equal superposition can 


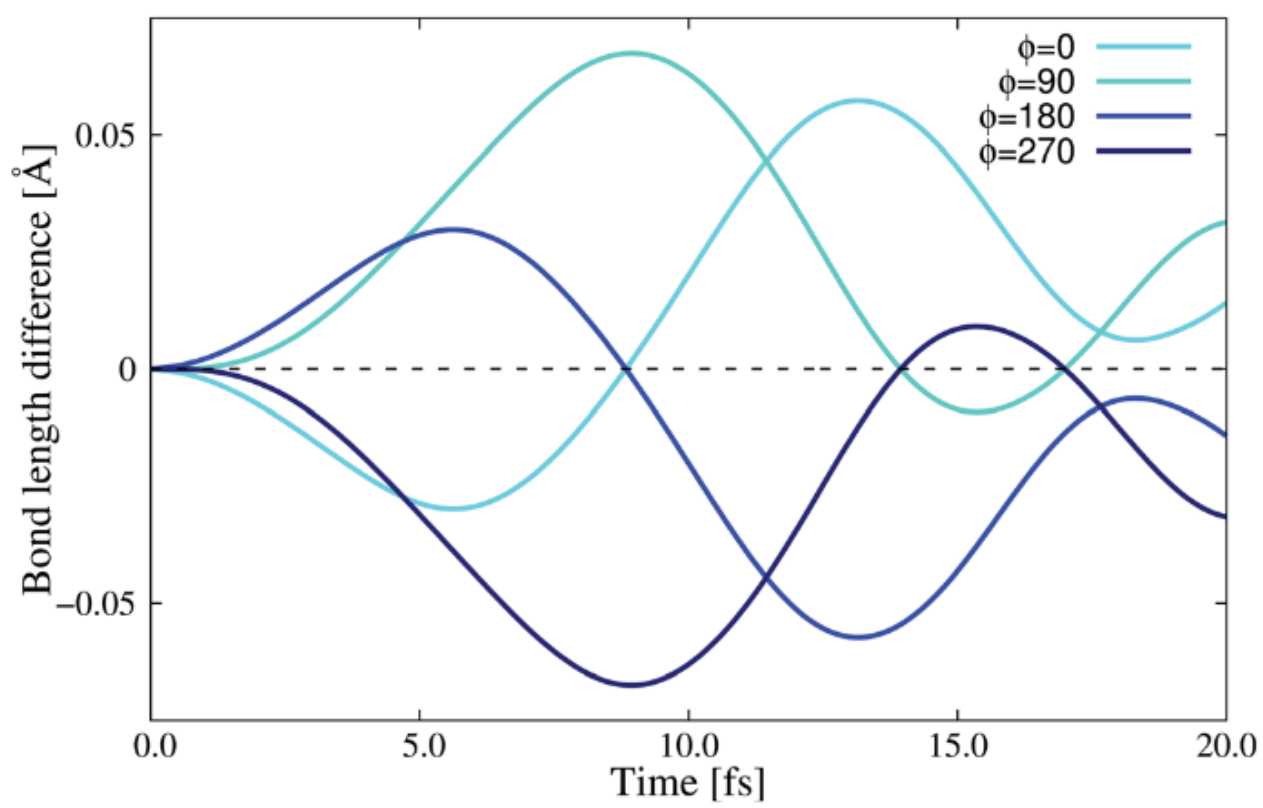

Figure 5: Time evolution of the difference in bond length between two methylene groups in BMA[6,5] upon excitation to initial superpositions with relative phases of $\phi=0, \pi / 2, \pi$ and $3 \pi / 2$. Reproduced from [Vacher et al, Faraday Discuss. 2016, 194, 95-115] with permission from the Royal Society of Chemistry.

control the nuclear dynamics along the derivative coupling vector, see Figure 6 . Their results demonstrated that while the controllability of nuclear dynamics relies upon electronic coherence still being present at the CI, strong non-adiabatic coupling can preserve the electronic coherence. This hints that while the CI must still be close to the Frank-Condon point to ensure control, stronger coupling may allow for the CI to be further from the Frank-Condon point.

Recently, Robb et al. investigated the coupled electron and nuclear dynamics induced in benzene cation upon population of various coherent superpositions of the 8 lowest-energy electronic states. ${ }^{92}$ The simulations were performed using the Quantum Ehrenfest method, in reduced dimensionality taking into account 12 nuclear coordinates. They observed how different electronic superpositions lead to different bond vibrations, hinting at different fragmentation pathways (Figure 7).

What does the future of attochemistry look like? A number of theoretical and experi- 

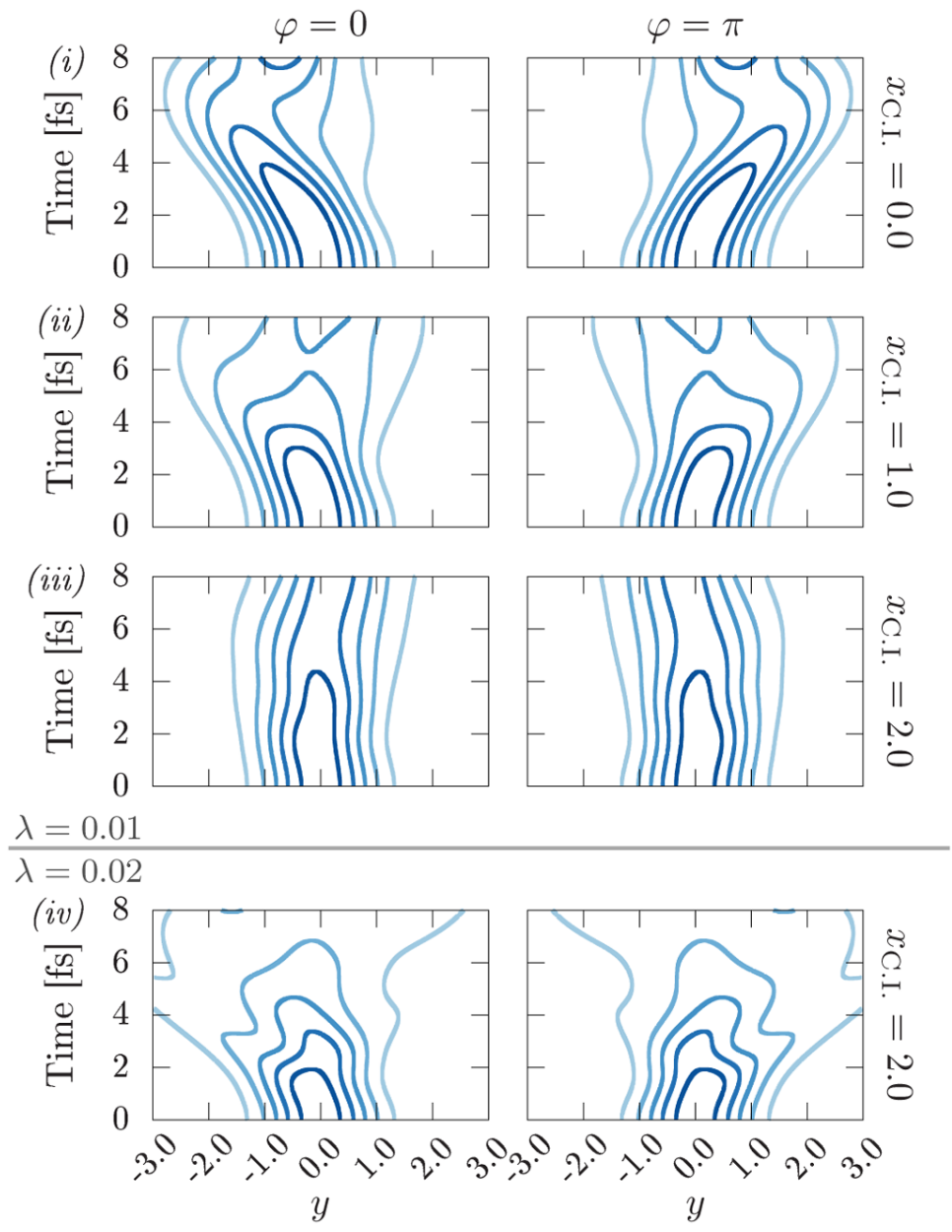

Figure 6: 1D nuclear density along the derivative coupling coordinate $y$ varying the relative phase $\phi$ imprinted on the electronic states, the nonadiabatic coupling strength $\lambda$, and the distance to the conical intersection $x_{\text {C.I. }}$. Reprinted figure with permission from [Arnold et al, Phys. Rev. Lett. 120, 123001 (2018)]. Copyright 2018 by the American Physical Society.

mental challenges have clearly emerged during the early investigations in the field. Early schemes proposed for the control of formation of electronic superpositions typically relied on CEP controlled IR pulses, ${ }^{9,29}$ due to the limitations of attosecond pulses at this time. ${ }^{35}$ Consequently, state-of-the-art theoretical studies have involved incorporation of CEP laser pulses into the already complex coupled electron-nuclear dynamics. ${ }^{46,54}$ The very first studies focused on the control of dissociation of diatomics upon ionisation, while recent studies aim at controlling the outcome (branching ratio) of a passage through a CI in more interesting molecular systems. Using this approach, the time it takes for the molecular wavepacket to 


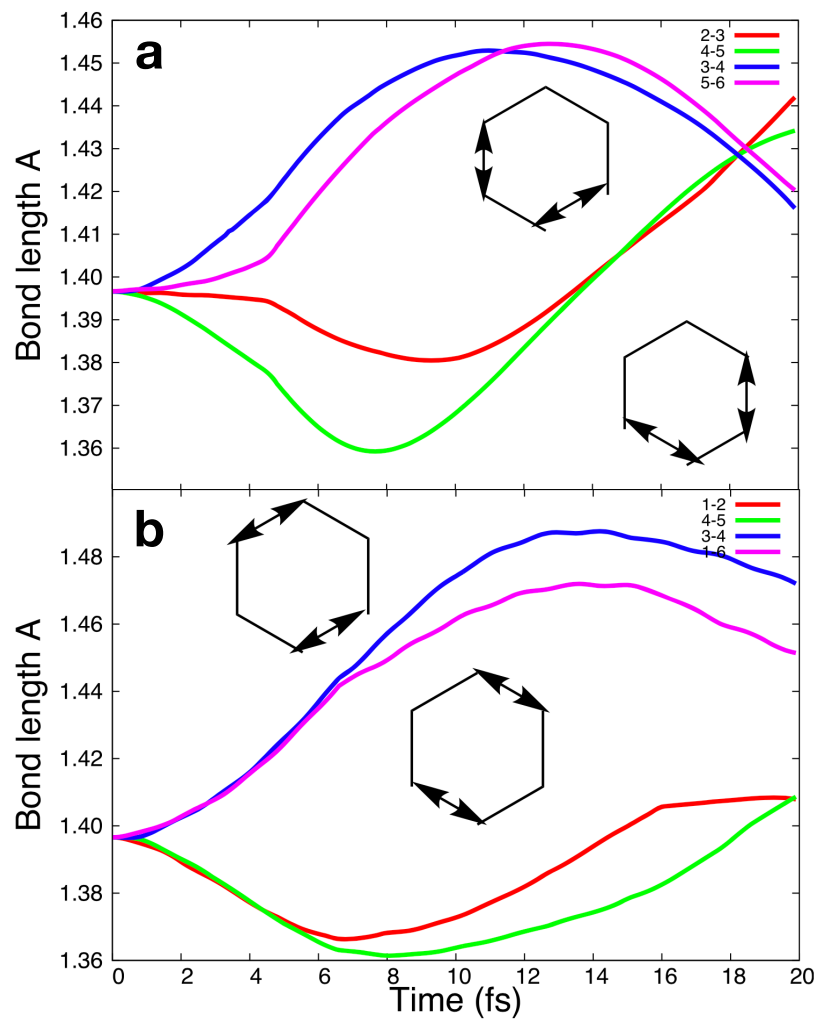

Figure 7: Bond vibrations induced in benzene cation upon population of coherent superpositions of (a) E and B, and (b) D and B electronic states. ${ }^{92}$

reach the CI must be well known in order to determine when to apply the IR pulse to build up the superposition. In addition, the effect of the IR pulse on the electron dynamics itself must also be considered. Strong field IR pulses can have significant effects on potential energy surfaces, and it can be difficult to determine if the final results originate in the electron dynamics due to the complex superposition itself, or to the alteration of the dynamics by the pulse itself. ${ }^{38,41,42}$ On the other hand, as suggested by a number of groups, ${ }^{46,47,54,71}$ the altering of the potential energy surfaces and dynamics by the IR pulse is another possible route to control the electron dynamics and reactivity. Finally, using IR pulses to control or induce CIs in molecules is another potential route to light-induced control of molecular reactions, which could be combined with attosecond schemes. ${ }^{9,93-96}$

Using attosecond pulses to generate specific electronic wavepackets and therefore trigger the desired electron dynamics, allowing for control of consequent nuclear motion is an 
appealing alternative proposal. ${ }^{21,71}$ It was first inspired by the initial experiments on photoionization, ${ }^{26,27}$ and is now also supported both by more recent measurements ${ }^{34}$ and theoretical studies. ${ }^{48-50,63}$ More precisely, the dependency of motion in the branching space on the initial superposition was demonstrated: through modification of the ratio and phase difference between the two states in the initial electronic superposition - altering $\theta / 2$ and $\phi$ respectively in Eq. (2) - both the direction and velocity of the initial nuclear dynamics after passage through the CI could be controlled. This approach may however be limited to systems having CIs close to the Franck-Condon region to outrun electronic decoherence. In addition, exciting directly to a phase-defined superposition of states is not so well theoretically explored as using the CEP of an IR control pulse to build up a superposition.

A large amount of theoretical and experimental work remains to be done however before "true" attochemistry - charge-directed reactivity achieved by direct control of electrons in a system - can be realized (Figure 8). First, reasoning "backward", a large amount of theoretical work remains to be done to reach a really trustworthy description of coupled electron-nuclear dynamics in polyatomic molecules. Simulations of chemical reactions controlled by an electronic wavepacket have been limited so far to diatomics or triatomics. Theoretical studies on larger molecular systems have only demonstrated the effect of electronic wavepackets on early nuclear motion and slight bond stretches rather than determined the final photochemical product. This is partly due to the current limitations of the existing theoretical methods. ${ }^{89}$ If the most accurate quantum nuclear dynamics simulations are preferable, they are often hampered by the exponential scaling of the calculation cost with respect to the number of nuclear degrees of freedom. Grid-based quantum dynamics simulations have often been limited to few nuclear coordinates. Using the MCTDH method, one can in principle consider more nuclear coordinates but the method still involves the development of a model Hamiltonian and, typically, a reduced number of nuclear coordinates. ${ }^{50}$ One can question these approximations, in particular the reduced dimensionality can bias the obtained control of nuclear motion in polyatomic molecules. ${ }^{96}$ In addition, the resort 
to a fitted model often based on harmonic potentials further increases the deviation from the real system, not only quantitatively but in some cases also qualitatively: while harmonic potentials are well suited to describe photophysical processes, these are not made to describe chemical reactions like dissociation or cis-trans isomerisation. "Direct" or "on-the-fly" dynamic methods, such as the semiclassical Ehrenfest method, circumvent these problems with nuclear trajectories taking into account all nuclear coordinates and propagated on the real potential energy surfaces. The limits of these direct methods for non-adiabatic dynamics are the classical treatment of the motion of nuclei and the non-exact description of electronic coherence. Given that the new distribution of electrons of an electronic wavepacket comes from interference between the different electronic eigenstates, it is crucial to describe accurately electronic (de)coherence. An excellent compromise is provided by the DD-vMCG method, based on the propagation of variationally coupled Gaussian wavepackets following quantum trajectories. The evolution of not only the wavepacket expansion coefficients, but also of the mean position and momentum of every Gaussian basis function is determined by the time-dependent Schrödinger equation. This method, which involves a quantum treatment of both electronic and nuclear coordinates, and their coupling, while retaining the advantages of a direct method, has already been applied to attosecond electron dynamics. ${ }^{62}$ One drawback of this method is the non-trivial diabatisation procedure: the electronic structure data calculated along the dynamics in the adiabatic basis needs to be transformed to a diabatic basis in which the quantum dynamics is simulated. Another difficulty is to reach convergence both in terms of electronic database and number of Gaussian basis functions. We underline that there exist several other variants of on-the-fly coupled Gaussian trajectory-based methods that are in principle quantum mechanically exact: in particular, the Quantum-Ehrenfest method of Robb was already applied in the present context. ${ }^{54}$ However, this method uses classical trajectories as basis, which does not guarantee convergence to the exact result. Next, once such complex coupled electron and nuclear dynamics allows for the identification of the optimal electronic wavepacket that leads to the desired chemical reactivity, the mod- 
elling of the formation of electronic wavepackets after excitation using realistic attosecond pulses is crucial to fully open up the possibility of control of electron dynamics. An accurate description of how complex pulses interact with real molecular systems is necessary to identify the optimal attosecond pulse. Finally, work remains to be done both theoretically and experimentally on the generation of attosecond pulses. Investigation of methods of designing and tailoring pulses, in order to create specific electronic wavepackets, will undoubtedly require collaboration between experimentalists and theoreticians.

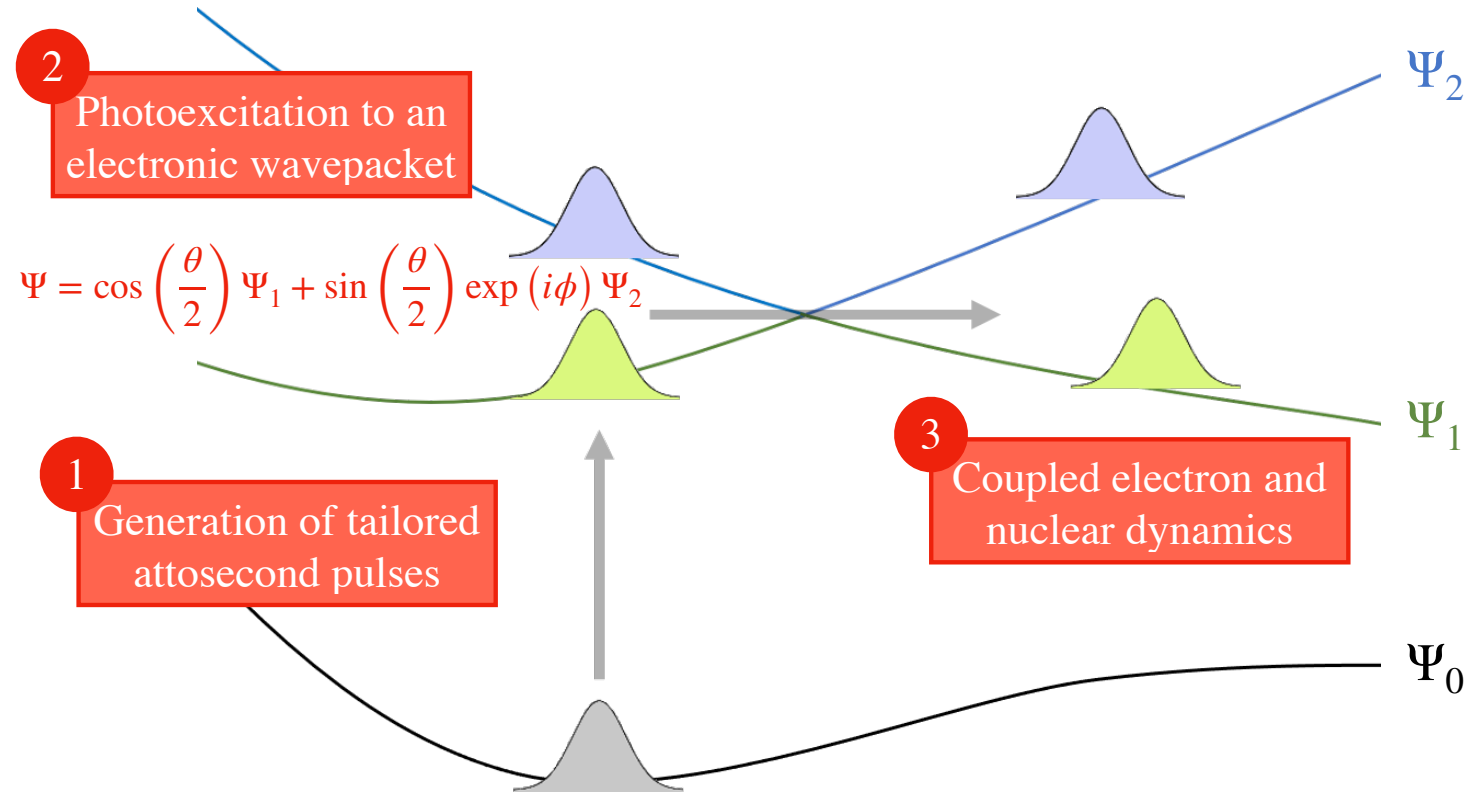

Figure 8: Scheme for attochemistry: excitation to a superposition of electronic states with defined population ratio and phase, using a conical intersection as the dynamical "probe" event to re-couple the states and direct the reactivity. Challenges moving forward for both theory and experiment are highlighted (red boxes).

The field of attochemistry remains in its infancy, 20 years on from the first generation of attosecond pulses. Nevertheless, much like how the advent of femtosecond pulses matching the timescale of nuclear dynamics eventually led to the now well-established field of femtochemistry allowing for steering of nuclei using femtosecond pulses, attosecond pulses open a route to directly control the motion of electrons inside molecules, which naturally occurs on an attosecond timescale. There is certainly a long way left to go, but the increasingly rapid developments on both theoretical and experimental fronts bring us closer to achieving 
"true" attochemistry.

\section{Acknowledgement}

I.M. acknowledges thesis funding from the University of Nantes. M.V. acknowledges the Région des Pays de la Loire for financial support through the framework of the PULSAR programme.

\section{References}

(1) Sibbett, W.; Lagatsky, A. A.; Brown, C. T. A. The Development and Application of Femtosecond Laser Systems. Opt. Express 2012, 20, 6989-7001.

(2) Shapiro, M.; Brumer, P. On the Origin of Pulse Shaping Control of Molecular Dynamics. J. Phys. Chem. A 2001, 105, 2897-2902.

(3) Brumer, P.; Shapiro, M. One Photon Mode Selective Control of Reactions by Rapid or Shaped Laser Pulses: An Emperor without Clothes? Chem. Phys. 1989, 139, 221-228.

(4) Charron, E.; Giusti-Suzor, A.; Mies, F. H. Coherent Control of Photodissociation in Intense Laser Fields. J. Chem. Phys. 1995, 103, 7359-7373.

(5) Tannor, D. J.; Kosloff, R.; Rice, S. A. Coherent Pulse Sequence Induced Control of Selectivity of Reactions: Exact Quantum Mechanical Calculations. J. Chem. Phys. 1986, 85, 5805-5820.

(6) Assion, A.; Baumert, T.; Bergt, M.; Brixner, T.; Kiefer, B.; Seyfried, V.; Strehle, M.; Gerber, G. Control of Chemical Reactions by Feedback-Optimized Phase-Shaped Femtosecond Laser Pulses. Science 1998, 282, 919-922.

(7) Brixner, T.; Damrauer, N. H.; Niklaus, P.; Gerber, G. Photoselective adaptive femtosecond quantum control in the liquid phase. Nature 2001, 414, 57-60. 
(8) Prokhorenko, V. I.; Nagy, A. M.; Waschuk, S. A.; Brown, L. S.; Birge, R. R.; Miller, R. J. D. Coherent Control of Retinal Isomerization in Bacteriorhodopsin. Science 2006, 313, 1257-1261.

(9) von den Hoff, P.; Thallmair, S.; Kowalewski, M.; Siemering, R.; de Vivie-Riedle, R. Optimal Control Theory - Closing the Gap between Theory and Experiment. Phys. Chem. Chem. Phys. 2012, 14, 14460.

(10) Nandipati, K. R.; Kanakati, A. K.; Singh, H.; Lan, Z.; Mahapatra, S. Initial StateSpecific Photodissociation Dynamics of Pyrrole via ${ }^{1} \pi \sigma^{*} / S_{0}$ Conical Intersection Initiated with Optimally Controlled UV-Laser Pulses. Eur. Phys. J. D 2017, 71, 222.

(11) Levine, B. G.; Martínez, T. J. Isomerization Through Conical Intersections. Ann. Rev. Phys. Chem. 2007, 58, 613-634.

(12) Klessinger, M. Conical Intersections and the Mechanism of Singlet Photoreactions. Angew. Chem. Int. Ed. 1995, 34, 549-551.

(13) Bernardi, F.; Olivucci, M.; Robb, M. A. Potential Energy Surface Crossings in Organic Photochemistry. Chem. Soc. Rev. 1996, 25, 321-328.

(14) Polli, D.; Altoè, P.; Weingart, O.; Spillane, K. M.; Manzoni, C.; Brida, D.; Tomasello, G.; Orlandi, G.; Kukura, P.; Mathies, R. A. et al. Conical Intersection Dynamics of the Primary Photoisomerization Event in Vision. Nature 2010, 467, 440443.

(15) Blancafort, L. Photochemistry and Photophysics at Extended Seams of Conical Intersection. ChemPhysChem 2014, 15, 3166-3181.

(16) Nenov, A.; Cordes, T.; Herzog, T. T.; Zinth, W.; de Vivie-Riedle, R. Molecular Driving Forces for Z/E Isomerization Mediated by Heteroatoms: The Example Hemithioindigo. J. Phys. Chem. A 2010, 114, 13016-13030. 
(17) Paul, P. M. Observation of a Train of Attosecond Pulses from High Harmonic Generation. Science 2001, 292, 1689-1692.

(18) Hentschel, M.; Kienberger, R.; Spielmann, C.; Reider, G. A.; Milosevic, N.; Brabec, T.; Corkum, P.; Heinzmann, U.; Drescher, M.; Krausz, F. Attosecond Metrology. Nature 2001, 414, 509-513.

(19) Orfanos, I.; Makos, I.; Liontos, I.; Skantzakis, E.; Förg, B.; Charalambidis, D.; Tzallas, P. Attosecond Pulse Metrology. APL Photonics 2019, 4, 080901.

(20) Krausz, F.; Ivanov, M. Attosecond Physics. Rev. Mod. Phys. 2009, 81, 163-234.

(21) Lépine, F.; Ivanov, M. Y.; Vrakking, M. J. J. Attosecond Molecular Dynamics: Fact or Fiction? Nature Photon 2014, 8, 195-204.

(22) Nisoli, M.; Decleva, P.; Calegari, F.; Palacios, A.; Martín, F. Attosecond Electron Dynamics in Molecules. Chem. Rev. 2017, 117, 10760-10825.

(23) Remacle, F.; Levine, R. D. An electronic time scale in chemistry. Proceedings of the National Academy of Sciences 2006, 103, 6793-6798.

(24) Weitzel, K.-M. Controlling the Electrons Provides Means for Controlling Chemistry. ChemPhysChem 2007, 8, 213-215.

(25) Remacle, F.; Levine, R. D.; Ratner, M. A. Charge Directed Reactivity: A Simple Electronic Model, Exhibiting Site Selectivity, for the Dissociation of Ions. Chem. Phys. Lett. 1998, 285, 25-33.

(26) Weinkauf, R.; Schanen, P.; Metsala, A.; Schlag, E. W.; Bürgle, M.; Kessler, H. Highly Efficient Charge Transfer in Peptide Cations in the Gas Phase: Threshold Effects and Mechanism. J. Phys. Chem. 1996, 100, 18567-18585.

(27) Weinkauf, R.; Schlag, E. W.; Martinez, T. J.; Levine, R. D. Nonstationary Electronic States and Site-Selective Reactivity. J. Phys. Chem. A 1997, 101, 7702-7710. 
(28) Remacle, F.; Levine, R. D.; Schlag, E. W.; Weinkauf, R. Electronic Control of Site Selective Reactivity: A Model Combining Charge Migration and Dissociation. J. Phys. Chem. A 1999, 103, 10149-10158.

(29) Kling, M. F.; Siedschlag, C.; Verhoef, A. J.; Khan, J. I.; Schultze, M.; Uphues, T.; Ni, Y.; Uiberacker, M.; Drescher, M.; Krausz, F. et al. Control of Electron Localization in Molecular Dissociation. Science 2006, 312, 246-248.

(30) Kling, M. F.; von den Hoff, P.; Znakovskaya, I.; de Vivie-Riedle, R. (Sub-)Femtosecond Control of Molecular Reactions via Tailoring the Electric Field of Light. Phys. Chem. Chem. Phys. 2013, 15, 9448.

(31) Ray, D.; He, F.; De, S.; Cao, W.; Mashiko, H.; Ranitovic, P.; Singh, K. P.; Znakovskaya, I.; Thumm, U.; Paulus, G. G. et al. Ion-Energy Dependence of Asymmetric Dissociation of $\mathbf{D}_{\mathbf{2}}$ by a Two-Color Laser Field. Phys. Rev. Lett. 2009, 103, 223201.

(32) Cederbaum, L.; Zobeley, J. Ultrafast Charge Migration by Electron Correlation. Chem. Phys. Lett. 1999, 307, 205-210.

(33) Sansone, G.; Kelkensberg, F.; Pérez-Torres, J. F.; Morales, F.; Kling, M. F.; Siu, W.; Ghafur, O.; Johnsson, P.; Swoboda, M.; Benedetti, E. et al. Electron Localization Following Attosecond Molecular Photoionization. Nature 2010, 465, 763-766.

(34) Calegari, F.; Ayuso, D.; Trabattoni, A.; Belshaw, L.; De Camillis, S.; Anumula, S.; Frassetto, F.; Poletto, L.; Palacios, A.; Decleva, P. et al. Ultrafast Electron Dynamics in Phenylalanine Initiated by Attosecond Pulses. Science 2014, 346, 336-339.

(35) Leone, S. R.; McCurdy, C. W.; Burgdörfer, J.; Cederbaum, L. S.; Chang, Z.; Dudovich, N.; Feist, J.; Greene, C. H.; Ivanov, M.; Kienberger, R. et al. What Will It Take to Observe Processes in 'Real Time'? Nature Photon 2014, 8, 162-166. 
(36) Roudnev, V.; Esry, B. D.; Ben-Itzhak, I. Controlling $\mathrm{HD}^{+}$and $\mathrm{H}_{2}{ }^{+}$Dissociation with the Carrier-Envelope Phase Difference of an Intense Ultrashort Laser Pulse. Phys. Rev. Lett. 2004, 93, 163601.

(37) Bandrauk, A. D.; Chelkowski, S.; Nguyen, H. S. Attosecond localization of electrons in molecules. Int. J. Quantum Chem. 2004, 100, 834-844.

(38) von den Hoff, P.; Znakovskaya, I.; Kling, M.; de Vivie-Riedle, R. Attosecond Control of the Dissociative Ionization via Electron Localization: A Comparison between $\mathrm{D}_{2}$ and CO. Chem. Phys. 2009, 366, 139-147.

(39) Geppert, D.; von den Hoff, P.; de Vivie-Riedle, R. Electron Dynamics in Molecules: A New Combination of Nuclear Quantum Dynamics and Electronic Structure Theory. J. Phys. B: At. Mol. Opt. Phys. 2008, 41, 074006.

(40) Kling, M. F.; Vrakking, M. J. Attosecond Electron Dynamics. Annu. Rev. Phys. Chem. 2008, 59, 463-492.

(41) Znakovskaya, I.; von den Hoff, P.; Zherebtsov, S.; Wirth, A.; Herrwerth, O.; Vrakking, M. J. J.; de Vivie-Riedle, R.; Kling, M. F. Attosecond Control of Electron Dynamics in Carbon Monoxide. Phys. Rev. Lett. 2009, 103, 103002.

(42) Znakovskaya, I.; von den Hoff, P.; Schirmel, N.; Urbasch, G.; Zherebtsov, S.; Bergues, B.; de Vivie-Riedle, R.; Weitzel, K.-M.; Kling, M. F. Waveform Control of Orientation-Dependent Ionization of DCl in Few-Cycle Laser Fields. Phys. Chem. Chem. Phys. 2011, 13, 8653-8658.

(43) Korolkov, M. V.; Weitzel, K. M. Carrier Envelope Phase Effects in Photofragmentation: Orientation versus Alignment. Opt. Spectrosc. 2011, 111, 606.

(44) Cattaneo, P.; Persico, M. Wave Packet Dynamics in the Presence of a Conical Intersection. J. Phys. Chem. A 1997, 101, 3454-3460. 
(45) von den Hoff, P.; Siemering, R.; Kowalewski, M.; de Vivie-Riedle, R. Electron Dynamics and Its Control in Molecules: From Diatomics to Larger Molecular Systems. IEEE J. Sel. Top. Quantum Electron. 2012, 18, 119-129.

(46) Schüppel, F.; Schnappinger, T.; Bäuml, L.; de Vivie-Riedle, R. Waveform Control of Molecular Dynamics Close to a Conical Intersection. J. Chem. Phys. 2020, 153, 224307.

(47) Schnappinger, T.; de Vivie-Riedle, R. Coupled Nuclear and Electron Dynamics in the Vicinity of a Conical Intersection. J. Chem. Phys. 2021, 154, 134306.

(48) Vacher, M.; Meisner, J.; Mendive-Tapia, D.; Bearpark, M. J.; Robb, M. A. Electronic Control of Initial Nuclear Dynamics Adjacent to a Conical Intersection. J. Phys. Chem. A 2015, 119, 5165-5172.

(49) Meisner, J.; Vacher, M.; Bearpark, M. J.; Robb, M. A. Geometric Rotation of the Nuclear Gradient at a Conical Intersection: Extension to Complex Rotation of Diabatic States. J. Chem. Theory Comput. 2015, 11, 3115-3122.

(50) Arnold, C.; Vendrell, O.; Welsch, R.; Santra, R. Control of Nuclear Dynamics through Conical Intersections and Electronic Coherences. Phys. Rev. Lett. 2018, 120, 123001.

(51) Nikodem, A.; Levine, R. D.; Remacle, F. Spatial and Temporal Control of Populations, Branching Ratios, and Electronic Coherences in LiH by a Single One-Cycle Infrared Pulse. Phys. Rev. A 2017, 95, 053404.

(52) Ajay, J.; Šmydke, J.; Remacle, F.; Levine, R. D. Probing in Space and Time the Nuclear Motion Driven by Nonequilibrium Electronic Dynamics in Ultrafast Pumped N2. The Journal of Physical Chemistry A 2016, 120, 3335-3342, PMID: 26937745.

(53) Jenkins, A. J.; Spinlove, K. E.; Vacher, M.; Worth, G. A.; Robb, M. A. The Ehrenfest Method with Fully Quantum Nuclear Motion (Qu-Eh): Application to Charge Migration in Radical Cations. J. Chem. Phys. 2018, 149, 094108. 
(54) Tran, T.; Jenkins, A. J.; Worth, G. A.; Robb, M. A. The Quantum-Ehrenfest Method with the Inclusion of an IR Pulse: Application to Electron Dynamics of the Allene Radical Cation. J. Chem. Phys. 2020, 153, 031102.

(55) Ben-Nun, M.; Quenneville, J.; Martínez, T. J. Ab Initio Multiple Spawning: Photochemistry from First Principles Quantum Molecular Dynamics. The Journal of Physical Chemistry A 2000, 104, 5161-5175.

(56) Curchod, B. F. E.; Martínez, T. J. Ab Initio Nonadiabatic Quantum Molecular Dynamics. Chemical Reviews 2018, 118, 3305-3336, PMID: 29465231.

(57) Fabris, D.; Witting, T.; Okell, W. A.; Walke, D. J.; Matia-Hernando, P.; Henkel, J.; Barillot, T. R.; Lein, M.; Marangos, J. P.; Tisch, J. W. G. Synchronized Pulses Generated at $20 \mathrm{eV}$ and $90 \mathrm{eV}$ for Attosecond Pump-Probe Experiments. Nature Photon 2015, 9, 383-387.

(58) Fang, L.; Osipov, T.; Murphy, B. F.; Rudenko, A.; Rolles, D.; Petrovic, V. S.; Bostedt, C.; Bozek, J. D.; Bucksbaum, P. H.; Berrah, N. Probing Ultrafast Electronic and Molecular Dynamics with Free-Electron Lasers. J. Phys. B: At. Mol. Opt. Phys. 2014, 47, 124006.

(59) Kuleff, A. I.; Cederbaum, L. S. Radiation Generated by the Ultrafast Migration of a Positive Charge Following the Ionization of a Molecular System. Phys. Rev. Lett. 2011, 106, 053001.

(60) Kuleff, A. I.; Cederbaum, L. S. Ultrafast Correlation-Driven Electron Dynamics. J. Phys. B: At. Mol. Opt. Phys. 2014, 47, 124002.

(61) Kuleff, A. I.; Lünnemann, S.; Cederbaum, L. S. Ultrafast Charge Migration Following Valence Ionization of 4-Methylphenol: Jumping over the Aromatic Ring. J. Phys. Chem. A 2010, 114, 8676-8679. 
(62) Vacher, M.; Bearpark, M. J.; Robb, M. A.; Malhado, J. P. Electron Dynamics upon Ionization of Polyatomic Molecules: Coupling to Quantum Nuclear Motion and Decoherence. Phys. Rev. Lett. 2017, 118, 083001.

(63) Vacher, M.; Albertani, F. E. A.; Jenkins, A. J.; Polyak, I.; Bearpark, M. J.; Robb, M. A. Electron and Nuclear Dynamics Following Ionisation of Modified BismethyleneAdamantane. Faraday Discuss. 2016, 194, 95-115.

(64) Jenkins, A. J.; Vacher, M.; Bearpark, M. J.; Robb, M. A. Nuclear Spatial Delocalization Silences Electron Density Oscillations in 2-Phenyl-Ethyl-Amine (PEA) and 2-Phenylethyl-N,N-Dimethylamine (PENNA) Cations. J. Chem. Phys. 2016, 144 , 104110.

(65) Hassan, M. T.; Luu, T. T.; Moulet, A.; Raskazovskaya, O.; Zhokhov, P.; Garg, M.; Karpowicz, N.; Zheltikov, A. M.; Pervak, V.; Krausz, F. et al. Optical Attosecond Pulses and Tracking the Nonlinear Response of Bound Electrons. Nature 2016, 530, $66-70$.

(66) Galli, M.; Wanie, V.; Lopes, D. P.; Månsson, E. P.; Trabattoni, A.; Colaizzi, L.; Saraswathula, K.; Cartella, A.; Frassetto, F.; Poletto, L. et al. Generation of Deep Ultraviolet Sub-2-Fs Pulses. Opt. Lett. 2019, 44, 1308.

(67) Latka, T.; Shirvanyan, V.; Ossiander, M.; Razskazovskaya, O.; Guggenmos, A.; Jobst, M.; Fieß, M.; Holzner, S.; Sommer, A.; Schultze, M. et al. Femtosecond wavepacket revivals in ozone. Phys. Rev. A 2019, 99, 063405.

(68) Mignolet, B.; Levine, R. D.; Remacle, F. Charge Migration in the Bifunctional PENNA Cation Induced and Probed by Ultrafast Ionization: A Dynamical Study. J. Phys. B: At. Mol. Opt. Phys. 2014, 47, 124011.

(69) Nisoli, M. The Birth of Attochemistry. Opt. Photonics News 2019, 30, 32-39. 
(70) Palacios, A.; Martín, F. The Quantum Chemistry of Attosecond Molecular Science. WIREs Comput. Mol. Sci. 2020, 10, e1430.

(71) Golubev, N. V.; Kuleff, A. I. Control of Charge Migration in Molecules by Ultrashort Laser Pulses. Phys. Rev. A 2015, 91, 051401.

(72) Breidbach, J.; Cederbaum, L. S. Migration of Holes: Formalism, Mechanisms, and Illustrative Applications. J. Chem. Phys. 2003, 118, 3983-3996.

(73) Mendive-Tapia, D.; Vacher, M.; Bearpark, M. J.; Robb, M. A. Coupled ElectronNuclear Dynamics: Charge Migration and Charge Transfer Initiated near a Conical Intersection. J. Chem. Phys. 2013, 139, 044110.

(74) Born, M.; Huang, K. Dynamical Theory of Crystal Lattices; Clarendon press, 1954.

(75) Runge, E.; Gross, E. K. U. Density-Functional Theory for Time-Dependent Systems. Phys. Rev. Lett. 1984, 52, 997-1000.

(76) Casida, M. E.; Chong, D. Recent advances in density functional methods. Computational Chemistry: Reviews of Current Trends 1995,

(77) Schirmer, J. Beyond the random-phase approximation: A new approximation scheme for the polarization propagator. Phys. Rev. A 1982, 26, 2395-2416.

(78) Schirmer, J.; Trofimov, A. B. Intermediate state representation approach to physical properties of electronically excited molecules. The Journal of Chemical Physics 2004, 120, 11449-11464.

(79) Roos, B. O.; Taylor, P. R.; Siegbahn, P. E. A complete active space SCF method (CASSCF) using a density matrix formulated super-CI approach. Chemical Physics 1980, 48, $157-173$.

(80) Remacle, F.; Levine, R. D. Probing Ultrafast Purely Electronic Charge Migration in Small Peptides. Z. Phys. Chem. (Muenchen, Ger.) 2007, 221, 647-661. 
(81) Vacher, M.; Bearpark, M. J.; Robb, M. A. Oscillating Charge Migration between Lone Pairs Persists without Significant Interaction with Nuclear Motion in the Glycine and Gly-Gly-NH-CH 3 Radical Cations. J. Chem. Phys. 2014, 140, 201102.

(82) Vacher, M.; Mendive-Tapia, D.; Bearpark, M. J.; Robb, M. A. Electron Dynamics upon Ionization: Control of the Timescale through Chemical Substitution and Effect of Nuclear Motion. J. Chem. Phys. 2015, 142, 094105.

(83) Vacher, M.; Steinberg, L.; Jenkins, A. J.; Bearpark, M. J.; Robb, M. A. Electron Dynamics Following Photoionization: Decoherence Due to the Nuclear-Wave-Packet Width. Phys. Rev. A 2015, 92, 040502.

(84) Arnold, C.; Vendrell, O.; Santra, R. Electronic Decoherence Following Photoionization: Full Quantum-Dynamical Treatment of the Influence of Nuclear Motion. Phys. Rev. A 2017, 95, 033425 .

(85) Despré, V.; Golubev, N. V.; Kuleff, A. I. Charge Migration in Propiolic Acid: A Full Quantum Dynamical Study. Phys. Rev. Lett. 2018, 121, 203002.

(86) Csehi, A.; Badankó, P.; Halász, G. J.; Vibók, Á.; Lasorne, B. On the preservation of coherence in the electronic wavepacket of a neutral and rigid polyatomic molecule. $J$. Phys. B.: Mol. Opt. Phys. 2020, 53, 184005.

(87) Worth, G. A.; Meyer, H.-D.; Köppel, H.; Cederbaum, L. S.; Burghardt, I. Using the MCTDH Wavepacket Propagation Method to Describe Multimode non-Adiabatic Dynamics. Int. Rev. Phys. Chem. 2008, 27, 569-606.

(88) Richings, G.; Polyak, I.; Spinlove, K.; Worth, G.; Burghardt, I.; Lasorne, B. Quantum Dynamics Simulations using Gaussian Wavepackets: the vMCG Method. Int. Rev. Phys. Chem. 2015, 34, 269-308. 
(89) Vacher, M.; Bearpark, M. J.; Robb, M. A. Direct Methods for non-Adiabatic Dynamics: Connecting the Single-Set Variational multi-Configuration Gaussian (vMCG) and Ehrenfest Perspectives. Theor. Chem. Acc. 2016, 135, 1-11.

(90) Valentini, A.; van den Wildenberg, S.; Remacle, F. Selective bond formation triggered by short optical pulses: quantum dynamics of a four-center ring closure. Phys. Chem. Chem. Phys. 2020, 22, 22302-22313.

(91) Gonçalves, C. E. M.; Levine, R. D.; Remacle, F. Ultrafast geometrical reorganization of a methane cation upon sudden ionization: an isotope effect on electronic nonequilibrium quantum dynamics. Phys. Chem. Chem. Phys. 2021, 23, 12051-12059.

(92) Tran, T.; Worth, G. A.; Robb, M. A. Control of Nuclear Dynamics in the Benzene Cation by Electronic Wavepacket Composition. Commun. Chem. 2021, 4, 48.

(93) Richter, M.; Bouakline, F.; González-Vázquez, J.; Martínez-Fernández, L.; Corral, I.; Patchkovskii, S.; Morales, F.; Ivanov, M.; Martín, F.; Smirnova, O. Sub-Laser-Cycle Control of Coupled Electron-Nuclear Dynamics at a Conical Intersection. New J. Phys. 2015, 17, 113023.

(94) Demekhin, P. V.; Cederbaum, L. S. Light-Induced Conical Intersections in Polyatomic Molecules: General Theory, Strategies of Exploitation, and Application. J. Chem. Phys. 2013, 139, 154314.

(95) Arasaki, Y.; Wang, K.; McKoy, V.; Takatsuka, K. Monitoring the Effect of a Control Pulse on a Conical Intersection by Time-Resolved Photoelectron Spectroscopy. Phys. Chem. Chem. Phys. 2011, 13, 8681-8689.

(96) Fábri, C.; Lasorne, B.; Halász, G. J.; Cederbaum, L. S.; Vibók, Á. Quantum LightInduced Nonadiabatic Phenomena in the Absorption Spectrum of Formaldehyde: FullAnd Reduced-Dimensionality Studies. J. Chem. Phys. 2020, 153, 234302. 